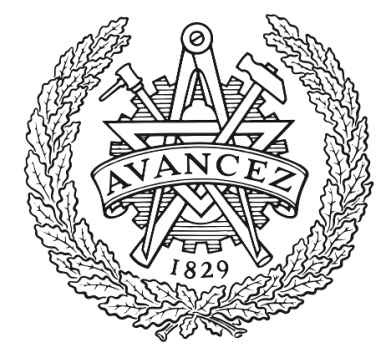

\title{
CHALMERS
}

UNIVERSITY OF TECHNOLOGY

\section{Localization Optimal Multi-user Beamforming with multi-carrier mmWave MIMO}

Downloaded from: https://research.chalmers.se, 2023-04-26 09:02 UTC

Citation for the original published paper (version of record):

Koirala, R., Denis, B., Uguen, B. et al (2018). Localization Optimal Multi-user Beamforming with multi-carrier mmWave MIMO. IEEE International Symposium on Personal, Indoor and Mobile Radio Communications, PIMRC: 1-7. http://dx.doi.org/10.1109/PIMRC.2018.8580712

N.B. When citing this work, cite the original published paper. 


\title{
Localization Optimal Multi-user Beamforming with multi-carrier mmWave MIMO
}

\author{
Remun Koirala* ${ }^{* \dagger}$, Benoît Denis*, Bernard Uguen ${ }^{\ddagger}$, Davide Dardari ${ }^{\dagger}$ and Henk Wymeersch $\S$ \\ ${ }^{*}$ CEA-Leti Minatec Campus, 17 rue des Martyrs, 38054 Grenoble Cedex 09, France \\ $\dagger$ DEI, University of Bologna, via Venezia 52, I-47521 Cesena (FC), Italy \\ $\ddagger$ University of Rennes 1-IETR (CNRS UMR 6164), Av. du General Leclerc, 35042, Rennes, France \\ $\S$ Department of Electrical Engineering, Chalmers University of Technology, Gothenburg, Sweden
}

\begin{abstract}
In this paper, we propose optimal beamforming strategies for a millimeter wave (mmWave) system consisting of multiple users based on the localization performance bounds. We consider a single base station (BS) with prior coarse knowledge of the users' positions and formulate the optimal beamforming problem in order to minimize the localization error consisting of Cramer Rao Lower Bounds (CRLBs) of delay, angle of departure (AoD) and angle of arrival (AoA) estimation at the mobile users. We first formulate the simplified CRLB of estimation parameters, taking advantage of multiple sub-carriers, and then formulate the localization error for optimization of the beamformer. Finally, we evaluate the resulting position and orientation error bounds after optimization for several fairness strategies through Monte Carlo simulations.
\end{abstract}

\section{INTRODUCTION}

The growing demand of device centric applications in wireless communication networks has induced the need for high precision location information [1]. In the context of next generation high frequency millimeter wave (mmWave) technologies, the use of highly directional and steerable antennas, to supplement the increasing high data rate and low latency requirements and contributing to counteract the severe path loss and high shadowing loss, has prompted for high positioning precision. In this context, the current state of the art satellite-based positioning systems such as Global Positioning System (GPS) or Galileo or the current wireless communication standards such as WiFi or Bluetooth based radio-location techniques are unreliable, especially in the urban and indoor scenarios [2]. Hence, high accuracy localization in the context of mmWave has recently become a topic of interest in the scientific community.

Initial works in this area were focused on characterizing the theoretical performance bounds in terms of position and orientation Cramer Rao Lower Bound (CRLB) for both a line-of-sight (LOS) single-path [3], and for a multi-path case [4], [5]. Similarly, the high spatial susceptibility due to high path loss and shadowing loss have inspired the community to understand and estimate the spatial channel between the transmitter and receiver. Using various signal processing tools such as compressed sensing and convex optimization, various channel estimation algorithms have been developed [5][8]. Finally, other contributions aim at optimizing the transmit beamforming so as to reduce the localization error. For instance, [9], [10] have used the theoretical bounds to optimize the beamformer for single and multiple sub-carriers respectively for single user case.

In this paper, we thus formulate and solve the optimal beamforming problem minimizing the localization error. To this aim, we first derive the expressions for the CRLBs of location-dependent estimation parameters (delay, angle of departure (AoD), angle of arrival (AoA)) taking advantage of multiple subcarriers. Then we formulate an equivalent localization error cost combining the previous bounds. Finally, we re-inject the optimization result in Position/Orientation Error bounds (PEB/OEB) and introduce two localization fairness strategies with respect to the different users.

\section{SYSTEM MODEL}

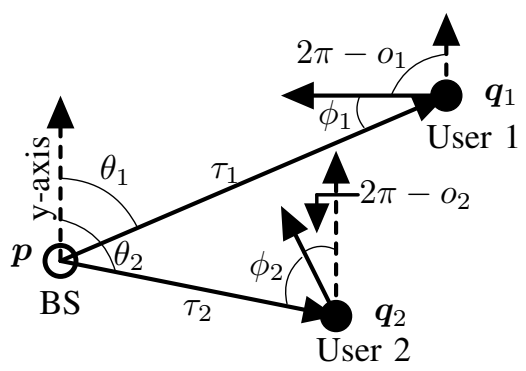

Fig. 1: Example of the system model with 1 BS and 2 users with orientations $o_{1}$ and $o_{2}$ at locations $\boldsymbol{q}_{1}$ and $\boldsymbol{q}_{2}$ respectively

Consider a mmWave down-link scenario consisting of a base station (BS) located at $\boldsymbol{p}=\left[p_{x}, p_{y}\right]^{T} \in \mathbb{R}^{2}$ and $U$ users, each user $u$ located at $\boldsymbol{q}_{u}=\left[q_{x, u}, q_{y, u}\right]^{T} \in \mathbb{R}^{2}$. Assume that the BS and each user is equipped with a uniform linear array (ULA) antenna with $N_{t}$ and $N_{r}$ antenna elements respective. Likewise, as illustrated in 
Fig. 1, the orientation of $u$-th user (relative to the y axis) is given by $\boldsymbol{o}_{u} \in[0,2 \pi)$.

The complex signal at a generic time instance is transmitted across $N$ subcarriers centered at frequency $f_{c}$ with bandwidth $B$ and is denoted by $s_{n}$ for the $n$-th subcarrier where $n \in\left[\frac{-N}{2}, \cdots, \frac{N}{2}\right]$. The individual elements of the set $n$ are denoted as $n_{i}$ where $i=1, \cdots N$.

Let $\boldsymbol{f}_{n} \in \mathbb{C}^{N_{t}}$ represent the beamformer (intended in a precoding sense) for the $n$-th subcarrier and we consider the beamforming power at each subcarrier $\left\|\boldsymbol{f}_{n}\right\|^{2} \leq 1$ [9], and the total beamforming power $\sum_{n}^{N}\left\|\boldsymbol{f}_{n}\right\|^{2}=K$. In this paper, we consider uniquely the direct path, assuming a LOS propagation model [9]. The $N_{t} \times N_{r}$ complex channel matrix (intended in a wide-sense, including antenna responses) for the $n$-th subcarrier between the BS and user- $u$ is denoted by $\boldsymbol{H}_{u, n}$ and is formulated as in [6].

$$
\boldsymbol{H}_{u, n}=\sqrt{\xi_{u}} h_{u} e^{-j 2 \pi \tau_{u} \frac{n B}{N}} \boldsymbol{a}_{R x, u}\left(\phi_{u}\right) \boldsymbol{a}_{T x, u}^{H}\left(\theta_{u}\right),
$$

where $h_{u} \in \mathbb{C}$ is the complex channel coefficient, $\xi_{u}$ is the path-loss between the BS and the user, and $\tau_{u}$, $\theta_{u}$ and $\phi_{u}$ are the delay, AoD and AoA respectively associated with user $u$. The transmit antenna response $\boldsymbol{a}_{T x, u}\left(\theta_{u}\right) \in \mathbb{C}^{N_{t}}$ can be expressed as

$$
\boldsymbol{a}_{T x, u}=\frac{1}{\sqrt{N_{t}}}\left[1, e^{j \frac{2 \pi}{\lambda} d \cos \left(\theta_{u}\right)}, \cdots, e^{j\left(N_{t}-1\right) \frac{2 \pi}{\lambda} d \cos \left(\theta_{u}\right)}\right]^{T} .
$$

For the ease of notation, here, we write $\boldsymbol{a}_{T x, u}\left(\theta_{u}\right)$ as $\boldsymbol{a}_{T x, u}$ and do the same for $\boldsymbol{a}_{R x, u}\left(\phi_{u}\right)$.

The receive antenna response $\boldsymbol{a}_{R x, u}$ can be expressed by simply replacing $\theta_{u}$ with $\phi_{u}$ and $N_{t}$ with $N_{r}$ in equation (2). We consider the post processed signal at the $u$-th user after whitening, which is denoted by $y_{u, n} \in \mathbb{C}$, as

$$
y_{u, n}=\sqrt{P_{T x, u}} \boldsymbol{w}_{u}^{H} \boldsymbol{H}_{u, n} \boldsymbol{f}_{n} s_{n}+\tilde{n}_{n},
$$

where, $P_{T x, u}$ is the transmitted power at the user and $\tilde{n}_{n} \in \mathbb{C}$ is the zero-mean additive white Gaussian noise (AWGN) with two-sided spectral density of $N_{0} / 2$ and $\boldsymbol{w}_{u} \in \mathbb{C}^{N_{r}}$ is the combiner vector at the mobile user.

\section{DERIVATION OF FISHER INFORMATION MATRIX (FIM) AND CRLB}

Here, we derive the FIM, firstly for the $n$-th subcarrier and then its generalization for all the sub-carriers, and then the CRLB for joint delay, AoD and AoA estimation.

\section{A. FIM}

Consider the estimation parameters $\boldsymbol{\eta}_{u}=$ $\left[\begin{array}{lllll}\tau_{u} & \theta_{u} & \phi_{u} & h_{R, u} & h_{I, u}\end{array}\right]$, where $h_{R, u}$ and $h_{I, u}$ represent respectively the real and imaginary parts of the complex channel coefficient. Then, the FIM $\boldsymbol{J}_{n, u} \in \mathbb{R}^{5 \times 5}$ for the estimation of the previous parameters for a given sub-carrier can be expressed [3], [4], [5], [9] as

$$
\boldsymbol{J}_{u, n}=\left[\begin{array}{ccccc}
\Phi_{n}\left(\tau_{u}, \tau_{u}\right) & \Phi_{n}\left(\tau_{u}, \theta_{u}\right) & \Phi_{n}\left(\tau_{u}, \phi_{u}\right) & \Phi_{n}\left(\tau_{u}, h_{R, u}\right) & \Phi_{n}\left(\tau_{u}, h_{I, u}\right) \\
\Phi_{n}\left(\theta_{u}, \tau_{u}\right) & \Phi_{n}\left(\theta_{u}, \theta_{u}\right) & \Phi_{n}\left(\theta_{u}, \phi_{u}\right) & \Phi_{n}\left(\theta_{u}, h_{R, u}\right) & \Phi_{n}\left(\theta_{u}, h_{I, u}\right) \\
\Phi_{n}\left(\phi_{u}, \tau_{u}\right) & \Phi_{n}\left(\phi_{u}, \theta_{u}\right) & \Phi_{n}\left(\phi_{u}, \phi_{u}\right) & \Phi_{n}\left(\phi_{u}, h_{R, u}\right) & \Phi_{n}\left(\phi_{u}, h_{I, u}\right) \\
\Phi_{n}\left(h_{R, u}, \tau_{u}\right) & \Phi_{n}\left(h_{R, u}, \theta_{u}\right) & \Phi_{n}\left(h_{R, u}, \phi_{u}\right) & \Phi_{n}\left(h_{R, u}, h_{R, u}\right) & \Phi_{n}\left(h_{R, u}, h_{I, u}\right) \\
\Phi_{n}\left(h_{I, u}, \tau_{u}\right) & \Phi_{n}\left(h_{I, u}, \theta_{u}\right) & \Phi_{n}\left(h_{I, u}, \phi_{u}\right) & \Phi_{n}\left(h_{I, u}, h_{R, u}\right) & \Phi_{n}\left(h_{I, u}, h_{I, u}\right)
\end{array}\right],
$$

where the values of the different matrix entries are given in appendix A. Now, the FIM in the multi-carrier case can be derived from equation (4) as follows.

$$
\boldsymbol{J}_{u}=\sum_{n=-N / 2}^{N / 2} \boldsymbol{J}_{u, n} .
$$

Considering the symmetry in the set $n$, and assuming $\boldsymbol{F}_{-n}=\boldsymbol{F}_{n}$ and $s_{-n}=s_{n}$ we can reformulate the FIM in a simple form. The components of FIM for multiple sub-carrier case can be found in appendix B.

\section{B. $C R L B$}

By considering the symmetric assumptions in the transmit signal, we significantly reduce the complexity of FIM inversion to derive the CRLB, even though we reduce the transmit diversity. By decoupling the estimation of delay parameter with the rest of the estimation parameters in the FIM in equation (5), we can simply invert the delay Fisher information to get the CRLB for delay estimation. For the remaining parameters, we can user Schur's complement as in [9] and find the CRLBs as follows.

$$
\begin{aligned}
\boldsymbol{J}_{\tau, u}^{-1} & =\frac{\alpha_{\tau, u}}{\boldsymbol{a}_{T x, u}^{H} \boldsymbol{X}_{\tau} \boldsymbol{a}_{T x, u}}, \\
\boldsymbol{J}_{\theta, u}^{-1} & =\frac{\alpha_{\theta, u}}{\left(\dot{\boldsymbol{a}}_{T x, u}^{H} \boldsymbol{X} \dot{\boldsymbol{a}}_{T x, u}-\frac{\left|\dot{\boldsymbol{a}}_{T x, u}^{H} \boldsymbol{X} \boldsymbol{a}_{T x, u}\right|^{2}}{\boldsymbol{a}_{T x, u}^{H} \boldsymbol{X} \boldsymbol{a}_{T x, u}}\right)}, \\
\boldsymbol{J}_{\phi, u}^{-1} & =\frac{\alpha_{\phi, u}}{\boldsymbol{a}_{T x, u}^{H} \boldsymbol{X} \boldsymbol{a}_{T x, u}} .
\end{aligned}
$$

where, $\alpha_{\tau, u}=\frac{1}{4 \pi^{2} \sigma_{u} d_{0, u}\left|h_{u}\right|^{2}}, \alpha_{\theta, u}=\frac{1}{d_{0, u} \sigma_{u}\left|h_{u}\right|^{2}}$, and $\alpha_{\phi, u}=\frac{1}{\sigma_{u}\left|h_{u}\right|^{2}\left(d_{2, u}-\frac{d_{1, u}^{2}}{d_{0, u}}\right)}$.

The variables $\boldsymbol{X}_{\tau}, \boldsymbol{X}, \dot{\boldsymbol{a}}_{T x, u}, \sigma_{u}$ and $d_{i, u}$ for $i \in$ $\{0,1,2\}$ are defined in appendices $\mathrm{A}$ and $\mathrm{B}$.

\section{LOCALIZATION ERROR OPTIMAL BEAMFOMER}

Taking the CRLB of delay, AoD and AoA into consideration from (6a) to (6c), we now define the overall localization error as a weighted sum, which can be expressed for each user $u$ as follows

$$
L_{u}=\gamma \beta_{\tau} J_{\tau, u}^{-1}+\beta_{\theta} J_{\theta, u}^{-1}+\beta_{\phi} J_{\phi, u}^{-1},
$$

where, $\gamma$ is the unit conversion factor from seconds to radians in order to maintain the homogeneity of the equation. Likewise, $\beta_{\tau}, \beta_{\theta}, \beta_{\phi} \in \mathbb{R}[0,1]$ represent the weights given to each estimation variable, namely delay, AoD, AoA respectively. The goal is to find the beamformer that minimizes this localization error. With the formulation in equation (7), we can analyze the 
effect of each estimation parameter independently onto beamforming by adjusting the weights.

We can however notice that there are two different variables $\boldsymbol{X}$ and $\boldsymbol{X}_{\tau}$ in the formulation of localization error, in (7). In order to consider a unique optimization variable, we can restructure the equation as follows.

Let $M=N_{t} \times N$. Then we define the vectors $\boldsymbol{a}_{u}$, $\dot{\boldsymbol{a}}_{u}$ and $\boldsymbol{a}_{N, u} \in \mathbb{C}^{M \times 1}$ as

$$
\begin{aligned}
& \boldsymbol{a}_{u}= {\left[\begin{array}{c}
\left|s_{n_{1}}\right| \boldsymbol{a}_{T x, u} \\
\left|s_{n_{2}}\right| \boldsymbol{a}_{T x, u} \\
\vdots \\
\left|s_{n_{N}}\right| \boldsymbol{a}_{T x, u}
\end{array}\right], \boldsymbol{a}_{N, u}=\left[\begin{array}{c}
\left|s_{n_{1}}\right| n_{1}^{2} \boldsymbol{a}_{T x, u} \\
\left|s_{n_{2}}\right| n_{2}^{2} \boldsymbol{a}_{T x, u} \\
\vdots \\
\left|s_{n_{N}}\right| n_{N}^{2} \boldsymbol{a}_{T x, u}
\end{array}\right] } \\
& \dot{\boldsymbol{a}}_{u}=\left[\begin{array}{c}
\left|s_{n_{1}}\right| \dot{\boldsymbol{a}}_{T x, u} \\
\left|s_{n_{2}}\right| \dot{\boldsymbol{a}}_{T x, u} \\
\vdots \\
\left|s_{n_{N}}\right| \dot{\boldsymbol{a}}_{T x, u}
\end{array}\right]
\end{aligned}
$$

and, $\boldsymbol{X}_{N} \in \mathbb{C}^{M \times M}$ is defined as the block diagonal matrix consisting of the beamforming matrix $\boldsymbol{F}_{n}$ over each sub-carrier, expressed as

$$
\boldsymbol{X}_{N}=\left[\begin{array}{llll}
\boldsymbol{F}_{n_{1}} & & & \\
& \boldsymbol{F}_{n_{2}} & & \\
& & \ddots & \\
& & & \boldsymbol{F}_{n_{N}}
\end{array}\right] .
$$

Hence, the localization error can be reformulated as

$$
\begin{aligned}
L_{u}\left(\boldsymbol{X}_{N}\right) & =\gamma \frac{\beta_{\tau} \alpha_{\tau, u}}{\boldsymbol{a}_{N, u}^{H} \boldsymbol{X}_{N} \boldsymbol{a}_{N, u}} \\
& +\frac{\beta_{\theta} \alpha_{\theta, u}}{\left(\dot{\boldsymbol{a}}_{u}^{H} \boldsymbol{X}_{N} \dot{\boldsymbol{a}}_{u}-\frac{\left|\dot{\boldsymbol{a}}_{u}^{H} \boldsymbol{X}_{N} \boldsymbol{a}_{u}\right|^{2}}{\boldsymbol{a}_{u}^{H} \boldsymbol{X}_{N} \boldsymbol{a}_{u}}\right)}+\frac{\beta_{\phi} \alpha_{\phi, u}}{\boldsymbol{a}_{u}^{H} \boldsymbol{X}_{N} \boldsymbol{a}_{u}} .
\end{aligned}
$$

The goal now is to formulate an optimization problem which minimizes this localization error (as optimization cost) under power constraints. This problem can be formulated as:

$$
\begin{aligned}
& \min _{\boldsymbol{X}_{N}} L_{u}\left(\boldsymbol{X}_{N}\right), \\
& \text { subject to: } \\
& \operatorname{trace}\left(\mathcal{I}_{i}^{T} \boldsymbol{X}_{N} \mathcal{I}_{i}\right) \leq 1, \quad \forall i, \\
& \operatorname{trace}\left(\mathcal{I}_{i}^{T} \boldsymbol{X}_{N} \mathcal{I}_{i}\right) \geq 0, \quad \forall i, \\
& \mathcal{I}_{i}^{T} \boldsymbol{X}_{N} \mathcal{I}_{j}=\mathbf{0}_{N_{t}}, \quad \forall i, j: i \neq j, \\
& \operatorname{trace}\left(\boldsymbol{X}_{N}\right)=K, \\
& \boldsymbol{X}_{N} \succeq 0, \\
& \operatorname{rank}\left(\mathcal{I}_{i}^{T} \boldsymbol{X}_{N} \mathcal{I}_{i}\right)=1 \quad \forall i .
\end{aligned}
$$

where $i, j \in\{1,2, \cdots, N\}$ and $\mathbf{0}_{N_{t}} \in \mathbb{R}^{N_{t} \times N_{t}}$ represents the zero matrix sized $N_{t} \times N_{t}$ and $\mathcal{I}_{n} \in \mathbb{R}^{N_{t} \times M}$ represents a matrix consisting of identity matrix $\boldsymbol{I}_{N_{t}}$ of size $N_{t}$ in $n$-th block position and $\mathbf{0}_{N_{t}}$ in the rest of the block positions.

$$
\mathcal{I}_{n}=\underbrace{[\underbrace{\mathbf{0}_{N_{t}}}_{1} \underbrace{\mathbf{0}_{N_{t}}}_{2} \cdots \underbrace{\boldsymbol{I}_{N_{t}}}_{n} \cdots \underbrace{\mathbf{0}_{N_{t}}}_{N}}_{\mathrm{N} \text { total blocks }}]^{T}
$$

The constraints from equations (11b) and (11c) define the power constraint at each subcarrier as assumed in the system model. Likewise, equation (11d) enforces the block diagonality constraint in the beamforming matrix $\boldsymbol{X}_{N}$. Equation (11e) represents the total power constraint across all the sub-carriers and by the virtue of equation (11b), we know that $K \leq N$. Similarly, from the positive semidefinite structure of the individual blocks $\boldsymbol{F}_{n}$ in $\boldsymbol{X}_{N}$, we can conclude that $\boldsymbol{X}_{N}$ is positive semidefinite as well and the rank of each block is 1 .

The objective function here, however, is non convex. However, it is possible to reformulate it into a convex optimization problem by introducing different slack variables $\zeta_{\tau}, \zeta_{\theta}, \zeta_{\phi}$ and represent the problem as follows:

$$
\max _{\boldsymbol{X}_{N}, \zeta_{\theta}, \zeta_{\phi}, \zeta_{\tau}} \beta_{\theta} \zeta_{\theta}+\beta_{\phi} \zeta_{\phi}+\gamma \beta_{\tau} \zeta_{\tau}
$$

subject to:

$$
\begin{aligned}
& \frac{\boldsymbol{a}_{N, u}^{H} \boldsymbol{X}_{N} \boldsymbol{a}_{N, u}}{\alpha_{\tau, u}} \geq \zeta_{\tau} \\
& \frac{1}{\alpha_{\theta, u}}\left(\dot{\boldsymbol{a}}_{u}^{H} \boldsymbol{X}_{N} \dot{\boldsymbol{a}}_{u}-\frac{\left|\dot{\boldsymbol{a}}_{u}^{H} \boldsymbol{X}_{N} \boldsymbol{a}_{u}\right|^{2}}{\boldsymbol{a}_{u}^{H} \boldsymbol{X}_{N} \boldsymbol{a}_{u}}\right) \geq \zeta_{\theta} \\
& \frac{\boldsymbol{a}_{u}^{H} \boldsymbol{X}_{N} \boldsymbol{a}_{u}}{\alpha_{\phi, u}} \geq \zeta_{\phi} \\
& \text { (11b)-(11g) }
\end{aligned}
$$

Note that the constraints from equations (13b) and (13d) are affine. From [11] and appendix C, we can simplify and cast the hyperbolic constraint in equation (13c) as a second order conic (SOC) constraint as:

$$
\begin{aligned}
& \left\|\left[\begin{array}{c}
2 \operatorname{Re}\left(\boldsymbol{a}_{u}{ }^{H} \boldsymbol{X}_{N} \boldsymbol{a}_{u}\right) \\
2 \operatorname{Im}\left(\boldsymbol{a}_{u}{ }^{H} \boldsymbol{X}_{N} \boldsymbol{a}_{u}\right) \\
\dot{\boldsymbol{a}}_{u}^{H} \boldsymbol{X}_{N} \dot{\boldsymbol{a}}_{u}-\zeta_{\theta} \alpha_{\theta, u}-\boldsymbol{a}_{u}^{H} \boldsymbol{X}_{N} \boldsymbol{a}_{u}
\end{array}\right]\right\|_{2} \\
& \leq \dot{\boldsymbol{a}}_{u}^{H} \boldsymbol{X}_{N} \dot{\boldsymbol{a}}_{u}-\zeta_{\theta} \alpha_{\theta, u}+\boldsymbol{a}_{u}^{H} \boldsymbol{X}_{N} \boldsymbol{a}_{u} .
\end{aligned}
$$

The objective function and all the constraints in equation (13), except the rank constraint in equation (11g), are convex. In order to solve this problem, in the literature, it is common to solve firstly the problem after dropping the incriminated constraint. Then, based on the first step optimization result, one gets the best rank-1 approximation for the matrix of interest. Hence, replacing the constraint in equation (13c) with equation (14) and removing the rank constraint, we can solve the convex semidefinite problem with efficient solvers [12].

Similarly, for a multi-user case, we define the localization error as the sum of localization errors per user.

$$
L\left(\boldsymbol{X}_{N}\right)=\sum_{u=1}^{U} L_{u}\left(\boldsymbol{X}_{N}\right)
$$


For the multi-user scenario, we also consider different resource allocation schemes based on different fairness criteria in order to distribute power to different users based on their positions with the help of beamforming optimization in order to minimize the localization error.

1) Min Max Fairness Strategy: In this strategy, we ensure a minimum localization error requirement for each user. In doing so, we are limited by the worst user, hence the optimal solution would lead to the minimization of the localization error of the user with maximum error. The optimization problem is thus formulated accordingly, as follows:

$$
\max _{\boldsymbol{X}_{N}, \zeta_{\theta}, \zeta_{\phi}, \zeta_{\tau}} \beta_{\theta} \zeta_{\theta}+\beta_{\phi} \zeta_{\phi}+\gamma \beta_{\tau} \zeta_{\tau}
$$

subject to:

$$
\begin{aligned}
& \frac{\boldsymbol{a}_{N, u}^{H} \boldsymbol{X}_{N} \boldsymbol{a}_{N, u}}{\alpha_{\tau, u}} \geq \zeta_{\tau}, \quad \forall u \\
& \left\|\left[\begin{array}{c}
2 R e\left(\dot{a}_{u}{ }^{H} \boldsymbol{X}_{N} \boldsymbol{a}_{u}\right) \\
2 \operatorname{Im}\left(\dot{\boldsymbol{a}}_{u}{ }^{H} \boldsymbol{X}_{N} \boldsymbol{a}_{u}\right) \\
\dot{\boldsymbol{a}}_{u}^{H} \boldsymbol{X}_{N} \dot{\boldsymbol{a}}_{u}-\zeta_{\theta} \alpha_{\theta, u}-\boldsymbol{a}_{u}^{H} \boldsymbol{X}_{N} \boldsymbol{a}_{u}
\end{array}\right]\right\|_{2} \\
& \leq \dot{\boldsymbol{a}}_{u}^{H} \boldsymbol{X}_{N} \dot{\boldsymbol{a}}_{u}-\zeta_{\theta} \alpha_{\theta, u}+\boldsymbol{a}_{u}^{H} \boldsymbol{X}_{N} \boldsymbol{a}_{u}, \quad \forall u \\
& \frac{\boldsymbol{a}_{u}^{H} \boldsymbol{X}_{N} \boldsymbol{a}_{u}}{\alpha_{\phi, u}} \geq \zeta_{\phi}, \quad \forall u
\end{aligned}
$$$$
\text { (11b)-(11g) }
$$

Note that the constraints in (16b)-(16d) the same CRLB requirement for each of the estimation parameters $\left(\zeta_{\tau}, \zeta_{\theta}\right.$ and $\left.\zeta_{\phi}\right)$ for each user. This condition serves to maximize the performance of the worst user.

2) Proportional Fairness Strategy: Alternatively, we can have a proportionally fair resource distribution strategy where better users receive proportionally more resources and hence have lower localization error compared to worse users. It has been shown that the diminishing returns property of the log function can be used to achieve proportional fairness [13]. The optimization problem can be written as

$$
\max _{\boldsymbol{X}_{N}, \zeta_{\theta, u}, \zeta_{\phi, u}, \zeta_{\tau, u}} \sum_{u=1}^{U} \log \left(\beta_{\theta} \zeta_{u, \theta}+\beta_{\phi} \zeta_{u, \phi}+\gamma \beta_{\tau} \zeta_{u, \tau}\right)
$$

subject to:

$$
\begin{aligned}
& \frac{\boldsymbol{a}_{n, u}^{H} \boldsymbol{X}_{N} \boldsymbol{a}_{n, u}}{\alpha_{\tau, u}} \geq \zeta_{\tau, u}, \quad \forall u \\
& \left\|\left[\begin{array}{c}
2 \operatorname{Re}\left(\dot{\boldsymbol{a}}_{u}{ }^{H} \boldsymbol{X}_{N} \boldsymbol{a}_{u}\right) \\
2 \operatorname{Im}\left(\dot{\boldsymbol{a}}_{u}{ }^{H} \boldsymbol{X}_{N} \boldsymbol{a}_{u}\right) \\
\dot{\boldsymbol{a}}_{u}^{H} \boldsymbol{X}_{N} \dot{\boldsymbol{a}}_{u}-\zeta_{\theta, u} \alpha_{\theta, u}-\boldsymbol{a}_{u}^{H} \boldsymbol{X}_{N} \boldsymbol{a}_{u}
\end{array}\right]\right\|_{2} \\
& \leq \dot{\boldsymbol{a}}_{u}^{H} \boldsymbol{X}_{N} \dot{\boldsymbol{a}}_{u}-\zeta_{\theta, u} \alpha_{\theta, u}+\boldsymbol{a}_{u}^{H} \boldsymbol{X}_{N} \boldsymbol{a}_{u} \quad \forall u(17 \mathrm{c}) \\
& \frac{\boldsymbol{a}_{u}^{H} \boldsymbol{X}_{N} \boldsymbol{a}_{u}}{\alpha_{\theta, u}} \geq \zeta_{\phi, u}, \quad \forall u \\
& \text { (11b)-(11g) }
\end{aligned}
$$

Since we solved the optimization problems in equations (13), (16) and (17) without the rank constraints to maintain convexity of the problem, we now approximate the rank-1 approximation of the matrix $\boldsymbol{F}_{n}^{*}$ as [14]:

$$
\boldsymbol{f}_{n}^{*}=\sqrt{\lambda_{n}} \boldsymbol{v}_{n},
$$

where $\lambda_{n}$ is the largest eigenvalue of each block element $\boldsymbol{F}_{n}^{*}$ of $\boldsymbol{X}_{N}^{*}$ and $\boldsymbol{v}_{n}$ is the corresponding eigenvector.

\section{Position AND ORIENTATION ERROR Bounds}

Even though we use delay, AoD and AoA as the estimation variables, PEB and OEB provide more intuitive understanding of the localization error of the user. Here, we introduce PEB and OEB which we can extract from the FIM in equation (5). Let $\boldsymbol{\mu}_{u}=$ $\left[p_{x, u}, p_{y, u}, \alpha_{u}, h_{r, u}, h_{i, u}\right]$ be the vector comprising of the new estimation variables representing the position in 2-D Cartesian plane, orientation and real and imaginary channel coefficients respectively. Similar to [5], [15], the FIM in terms of the new parameter can be written as

$$
\boldsymbol{J}_{\mu, u}=\boldsymbol{T}_{u} \boldsymbol{J}_{u}\left(\boldsymbol{X}_{N}\right) \boldsymbol{T}_{u}^{T},
$$

where $\boldsymbol{T}_{u}$ is the Jacobian of $\boldsymbol{\mu}_{u}$ with respect to the original estimation variables in $\boldsymbol{\eta}_{u}$ given by

$$
\boldsymbol{T}_{u}=\left[\begin{array}{ccccc}
\frac{\cos \left(\theta_{u}\right)}{c}\left(\begin{array}{cc}
c \\
c
\end{array}\right. & \frac{-\sin \left(\theta_{u}\right)}{\left\|\boldsymbol{p}_{u}-\boldsymbol{q}_{u}\right\|_{2}} & \frac{-\sin \left(\theta_{u}\right)}{\left\|\boldsymbol{p}_{u}-\boldsymbol{q}_{u}\right\|_{2}} & 0 & 0 \\
\frac{\sin \left(\theta_{u}\right)}{c} & \frac{\cos \left(\theta_{u}\right)}{\left\|\boldsymbol{p}_{u}-\boldsymbol{q}_{u}\right\|_{2}} & \frac{\cos \left(\theta_{u}\right)}{\left\|\boldsymbol{p}_{u}-\boldsymbol{q}_{u}\right\|_{2}} & 0 & 0 \\
0 & 0 & -1 & 0 & 0 \\
0 & 0 & 0 & 1 & 0 \\
0 & 0 & 0 & 0 & 1
\end{array}\right],
$$

where the constant $c$ is the speed of light.

Hence, we finally define PEB and OEB for user $u$ as:

$$
\begin{aligned}
\operatorname{PEB}_{u} & =\sqrt{\operatorname{trace}\left(\boldsymbol{J}_{\mu, u, 1: 2,1: 2}^{-1}\left(\boldsymbol{X}_{N}\right)\right)}, \\
\mathrm{OEB}_{u} & =\sqrt{\boldsymbol{J}_{\mu, u, 3,3}^{-1}\left(\boldsymbol{X}_{N}\right)} .
\end{aligned}
$$

\section{NUMERICAL RESULTS}

In this section we provide some illustrations of optimized beamformers in a canonical multi-user scenario.

\section{A. System Parameters and Setup}

Let us consider a mmWave BS operating at $f_{c}=38$ $\mathrm{GHz}$ with bandwidth $B=300 \mathrm{MHz}$. We fix the antenna elements number for both BS and user to $N_{t}=N_{r}=30$ elements. We consider both BS and user antennas to have a gain of $13 \mathrm{dBi}$ and an inter-element distance of $d=0.5 \lambda_{c}$. The path loss $\xi_{u}$ between the BS and any mobile user at a distance of $d_{u}$ from the BS is given as in [16].

$$
\xi_{u}\left(d_{u}\right)[d B]=\xi\left(d_{0}\right)+10 \alpha\left(d_{u} / d_{0}\right)+X_{\sigma},
$$

where, $\xi\left(d_{0}\right)$ is the free space path loss given by $10 \log _{10}\left(4 \pi d_{0} / \lambda_{c}\right)^{2}$ for a reference distance $d_{0}=1 \mathrm{~m}$ in our case. Parameters $\alpha=1.9$ is the path loss exponent and $\sigma=4.6$ is the standard deviation of the zero mean 


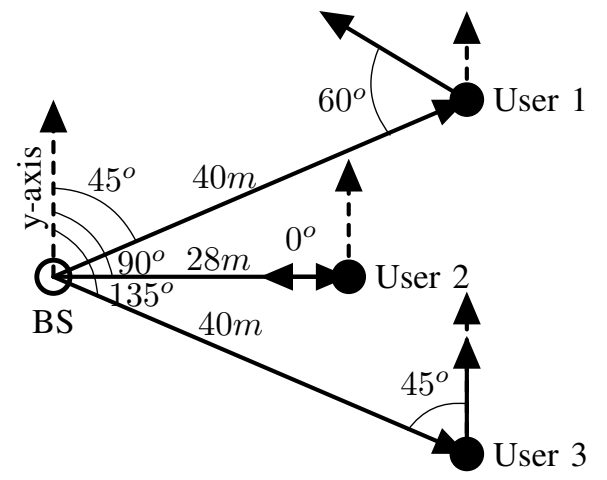

Fig. 2: Multi User Scenario

Relative beam gain for different strategies

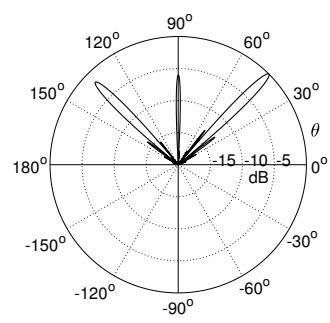

(a)

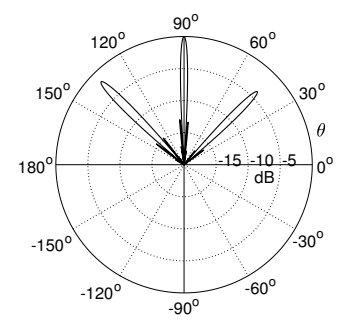

(b)
Fig. 3: Example of normalized beam direction for a localizationoptimized beamformer in the multi user case, according to (a) min max and (b) proportional fairness strategies

Gaussian random shadow factor $X_{\sigma}$ respectively. We consider the illustrating canonical scenario shown in Fig. 2 as the system model, unless otherwise specified.

\section{B. Results and Analysis}

In Figs. $3 a$ and $3 b$ we show the normalized beam gains as a function of BS transmission directions. We observe variable levels of power transmitted in the directions of the three distinct users depending on the fairness strategy. From Figs. 2, we observe that User 2 is the best user due to its proximity and orientation towards the base station followed by User 3 which is at the same distance as User 1, but with a different orientation. This is evident in Fig. 3 as with the min max fairness strategy, User 1 is allocated relatively more power compared to with the proportional fairness strategy.

In Fig. 4 and 5 we can see the effect of $\beta_{\tau}$ on the sub-carriers allocation. As discussed earlier, during localization, we consider symmetric power allocation across sub-carriers (with respect to the center frequency of the occupied spectrum) to facilitate the optimization problem we derived. Hence, in our analysis, we only consider the unique sub-carriers. We consider 8 subcarriers for simulation in this scenario, but only look at 4 of them numbered as $n=\{1,2,3,4\}$. We limit the total power $K$ to 2.5 units and optimize beamformer with $\beta_{\tau}=0$ and $\beta_{\tau}>0$. When $\beta_{\tau}>0$, we can observe

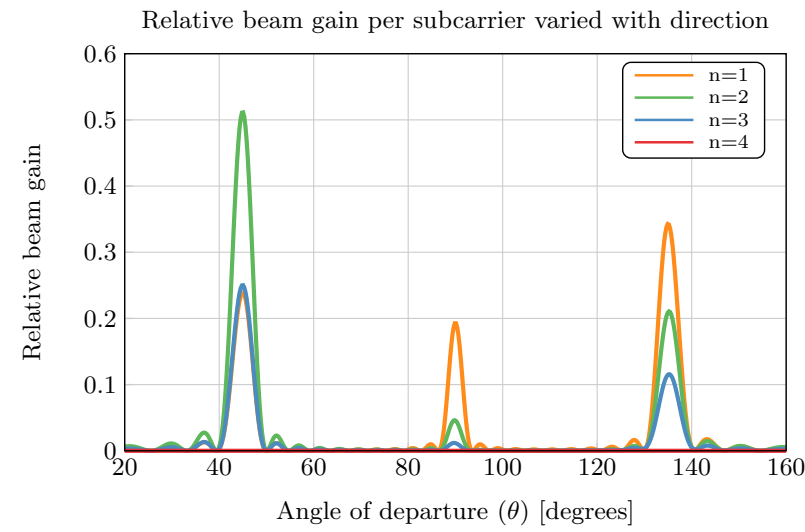

Fig. 4: Example of normalized beam gain (with respect to total gain) with min max fairness strategy as a function of direction for multi user case with $\beta_{\tau}=1, \beta_{\theta}=1$ and $\beta_{\phi}=1$

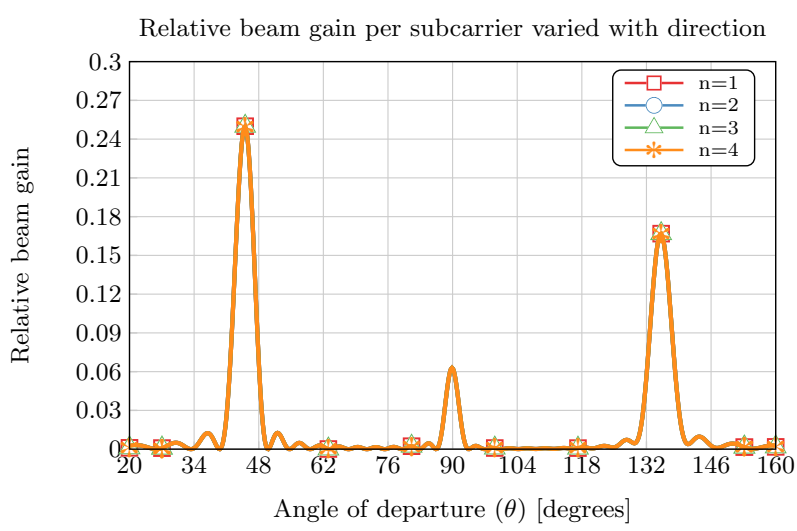

Fig. 5: Example of normalized beam gain (with respect to total gain) with min max fairness strategy as a function of direction for multi user case with $\beta_{\tau}=0, \beta_{\theta}=1$ and $\beta_{\phi}=1$

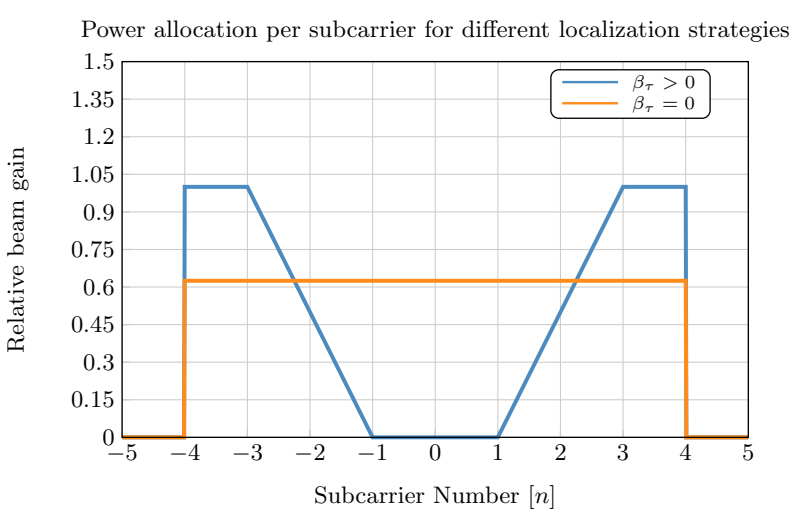

Fig. 6: Power allocation per sub-carrier for different values of $\beta_{\tau}$

that in Fig. 4, the first sub-carrier has no power allocated and the rest of the sub-carriers have unequal power distribution. However, for the other case where $\beta_{\tau}=0$, we have equal allocation across all the sub-carriers, as illustrated by Fig. 5. The sub-carrier power distribution for the two cases are presented in Fig. 6. It is clear that for $\beta_{\tau}>0$, the optimal beamformer would allocate all the power to the two extremities of the spectrum. 


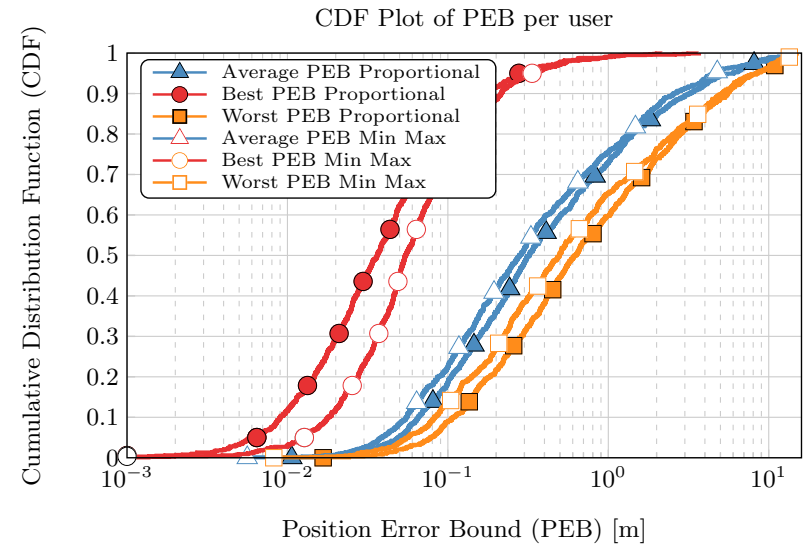

Fig. 7: Empirical CDF of the PEB per user (best, worst and average performance) for different fairness strategies over $1000 \mathrm{MC}$ trials.

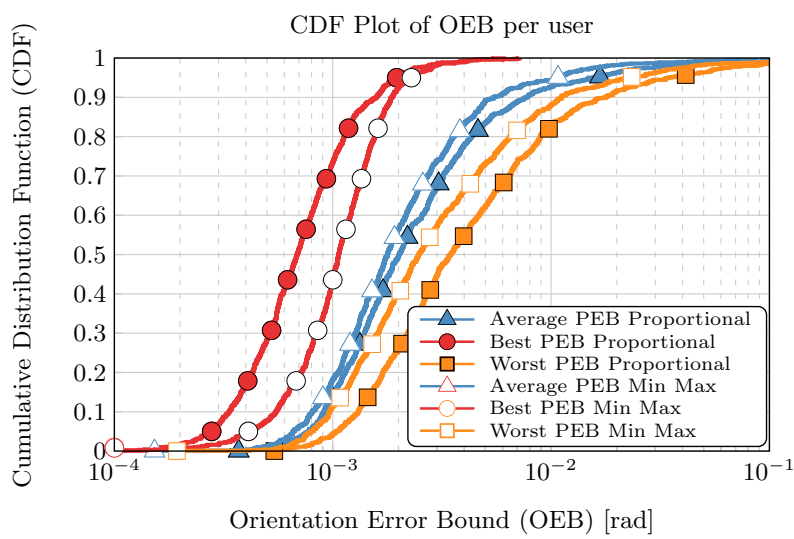

Fig. 8: Empirical CDF of the OEB per user (best, worst and average performance) for different fairness strategies over $1000 \mathrm{MC}$ trials.

The reason is that, for delay estimation, performance would benefit from higher resolution provided by a larger equivalent bandwidth (from using more distant frequency components). In contrast, for AoD and AoA estimation, since the frequency plays no role according to the underlying model, there is a uniform power allocation over all the sub-carriers.

Figs. 7 and 8 show the empirical cumulative distribution function (CDF) of best case, worst case and average PEB and OEB per user in the multi-user scenario, as a result of 1000 Monte Carlo (MC) simulation trials of the user positions consisting of the 3 random users positions/orientations (per trials) evaluated with both proportional and min max fairness strategies. In each occupied position of each MC trial, we characterize the best PEB as the one with minimum PEB, the worst PEB as the one with maximum PEB and the average PEB as the mean PEB over all three users (and similarly for OEB). We can observe that the CDFs of best, worst and average PEB and OEB are close to each other for all the cases. Even then, we can see that the proportional fairness, as expected, performs better for the best user whereas worse for the worst user and in average. It is also evident that the min max fairness improves the worst user performance, whereas the proportional fairness performs better improving the best user. Based on this, we can suggest that, if the difference is large between the worst and the best user, it is better to use the proportional fairness scheme such that the localization performance of the best user does not degrade too much whereas for a lower dispersion, min max optimization improves the overall performance more.

\section{CONCLUSIONS}

In this paper, we formulated and solved the multi user optimal beamforming problem minimizing a localization error cost, which combines the CRLBs characterizing the estimation errors of delay, AoD and AoA. In formulating the localization error, we derived the FIM and hence the CRLB for characterizing estimation error of intermediary location dependent parameters considering a multi carrier mmWave MIMO system. By tuning the $\beta \mathrm{s}$, we explored the effect of each localization estimation variable on the spectral power allocation. Finally, while optimizing the beamformer, we suggested two different strategies for power allocation, based on different definitions of fairness, to different users namely $\min \max$ and proportional fairness strategy, each one offering different resource allocation solution that can be adapted depending on the use case scenario.

\section{APPENDIX A}

\section{COMPONENTS OF THE FIM PER SUB-CARRIER}

Let $\boldsymbol{F}_{n}=\boldsymbol{f}_{n} \boldsymbol{f}_{n}^{H}$ and $\dot{\boldsymbol{a}}_{T x, u}=d \boldsymbol{a}_{T x, u} / d \theta$. The components of the FIM in equation (4), are as follows.

$$
\begin{aligned}
& \Phi_{n}\left(\tau_{u}, \tau_{u}\right)=4 \pi^{2} \rho_{u, n} \frac{n^{2} B^{2}}{N^{2}}\left|h_{u}\right|^{2} d_{0, u} \boldsymbol{a}_{T x, u}^{H} \boldsymbol{F}_{n} \boldsymbol{a}_{T x, u} \\
& \Phi_{n}\left(\tau_{u}, \theta_{u}\right)=2 \pi \rho_{u, n} \frac{n B}{N}\left|h_{u}\right|^{2} d_{0, u} \operatorname{Re}\left\{j \dot{\boldsymbol{a}}_{T x, u}^{H} \boldsymbol{F}_{n} \boldsymbol{a}_{T x, u}\right\} \\
& \Phi_{n}\left(\tau_{u}, \phi_{u}\right)=2 \pi \rho_{u, n} \frac{n B}{N}\left|h_{u}\right|^{2} d_{1, u} \operatorname{Re}\left\{j \boldsymbol{a}_{T x, u}^{H} \boldsymbol{F}_{n} \boldsymbol{a}_{T x, u}\right\} \\
& \Phi_{n}\left(\tau_{u}, h_{R, u}\right)=2 \pi \rho_{u, n} \frac{n B}{N} d_{0, u} \operatorname{Re}\left\{j h_{u}^{*} \boldsymbol{a}_{T x, u}^{H} \boldsymbol{F}_{n} \boldsymbol{a}_{T x, u}\right\} \\
& \Phi_{n}\left(\tau, h_{I, u}\right)=-2 \pi \rho_{u, n} \frac{n B}{N} d_{0, u} \operatorname{Re}\left\{h_{u}^{*} \boldsymbol{a}_{T x, u}^{H} \boldsymbol{F}_{n} \boldsymbol{a}_{T x, u}\right\} \\
& \Phi_{n}\left(\theta_{u}, \theta_{u}\right)=\rho_{u, n}\left|h_{u}\right|^{2} d_{0, u} \dot{\boldsymbol{a}}_{T x, u}^{H} \boldsymbol{F}_{n} \dot{\boldsymbol{a}}_{T x, u} \\
& \Phi_{n}\left(\theta_{u}, \phi_{u}\right)=\rho_{u, n}\left|h_{u}\right|^{2} \mathbb{R}\left\{d_{1, u} \boldsymbol{a}_{T x, u}^{H} \boldsymbol{F}_{n} \dot{\boldsymbol{a}}_{T x, u}\right\} \\
& \Phi_{n}\left(\theta_{u}, h_{R, u}\right)=\rho_{u, n} d_{0, u} \mathbb{R}\left\{h_{u} \dot{\boldsymbol{a}}_{T x, u}^{H} \boldsymbol{F}_{n} \boldsymbol{a}_{T x, u}\right\} \\
& \Phi_{n}\left(\theta_{u}, h_{I, u}\right)=\rho_{u, n} d_{0, u} \mathbb{I}\left\{h_{u} \dot{\boldsymbol{a}}_{T x, u}^{H} \boldsymbol{F}_{n} \boldsymbol{a}_{T x, u}\right\} \\
& \Phi_{n}\left(\phi_{u}, \phi_{u}\right)=\rho_{u, n}\left|h_{u}\right|^{2} d_{2, u} \boldsymbol{a}_{T x, u}^{H} \boldsymbol{F}_{n} \boldsymbol{a}_{T x, u} \\
& \Phi_{n}\left(\phi_{u}, h_{R, u}\right)=\rho_{u, n} \mathbb{R}\left\{h_{u} d_{1, u}\right\} \boldsymbol{a}_{T x, u}^{H} \boldsymbol{F}_{n} \boldsymbol{a}_{T x, u} \\
& \Phi_{n}\left(\phi_{u}, h_{I, u}\right)=\rho_{u, n} \mathbb{I}\left\{h_{u} d_{1, u}\right\} \boldsymbol{a}_{T x, u}^{H} \boldsymbol{F}_{n} \boldsymbol{a}_{T x, u} \\
& \Phi_{n}\left(h_{R, u}, h_{R, u}\right)=\rho_{u, n} d_{0, u} \boldsymbol{a}_{T x, u}^{H} \boldsymbol{F}_{n} \boldsymbol{a}_{T x, u} \\
& \Phi_{n}\left(h_{R, u}, h_{I, u}\right)=0 \\
& \Phi_{n}\left(h_{I, u}, h_{I, u}\right)=\rho_{u, n} d_{0, u} \boldsymbol{a}_{T x, u}^{H} \boldsymbol{F}_{n} \boldsymbol{a}_{T x, u} \\
& \text { where, } \rho_{u, n}=2 P_{T x, u} \xi_{u}\left|s_{n}\right|^{2} / N_{o} \text {, and } \\
& d_{0, u}=\left\|\boldsymbol{w}_{u}^{H} \boldsymbol{a}_{R x, u}\right\|_{2}^{2},
\end{aligned}
$$




$$
\begin{aligned}
& d_{1, u}=\boldsymbol{a}_{R x, u} \boldsymbol{w}_{u}^{H} \frac{d}{d \phi} \boldsymbol{w}_{u}^{H} \boldsymbol{a}_{R x, u}, \\
& d_{2, u}=\left\|\frac{d}{d \phi} \boldsymbol{w}_{u}^{H} \boldsymbol{a}_{R x, u}\right\|_{2}^{2} .
\end{aligned}
$$

\section{APPENDIX B}

\section{COMPONENTS OF THE FIM FOR ALL THE SUB-CARRIERS}

For the multiple sub-carrier case, we take advantage of the symmetry of the sub-carriers, beamformers, combiners and the data $s_{n}$ to formulate the FIM as follows.

$$
\begin{aligned}
& \Phi\left(\tau_{u}, \tau_{u}\right)=4 \pi^{2} \sigma_{u} \frac{B^{2}}{N^{2}}\left|h_{u}\right|^{2} d_{0, u} \boldsymbol{a}_{T x, u}^{H} \boldsymbol{X}_{\tau} \boldsymbol{a}_{T x, u} \\
& \Phi\left(\tau_{u}, \theta_{u}\right)=0 \\
& \Phi\left(\tau_{u}, \phi_{u}\right)=0 \\
& \Phi\left(\tau_{u}, h_{R, u}\right)=0 \\
& \Phi\left(\tau, h_{I, u}\right)=0 \\
& \Phi\left(\theta_{u}, \theta_{u}\right)=\sigma_{u}\left|h_{u}\right|^{2} d_{0, u} \dot{\boldsymbol{a}}_{T x, u}^{H} \boldsymbol{X} \dot{\boldsymbol{a}}_{T x, u} \\
& \Phi\left(\theta_{u}, \phi_{u}\right)=\sigma_{u}\left|h_{u}\right|^{2} \mathbb{R}\left\{d_{1, u} \boldsymbol{a}_{T x, u}^{H} \boldsymbol{X} \dot{\boldsymbol{a}}_{T x, u}\right\} \\
& \Phi\left(\theta_{u}, h_{R, u}\right)=\sigma_{u} d_{0, u} \mathbb{R}\left\{h_{u} \dot{\boldsymbol{a}}_{T x, u}^{H} \boldsymbol{X} \boldsymbol{a}_{T x, u}\right\} \\
& \Phi\left(\theta_{u}, h_{I, u}\right)=\sigma_{u} d_{0, u} \mathbb{I}\left\{h_{u} \dot{\boldsymbol{a}}_{T x, u}^{H} \boldsymbol{X} \boldsymbol{a}_{T x, u}\right\} \\
& \Phi\left(\phi_{u}, \phi_{u}\right)=\sigma_{u}\left|h_{u}\right|^{2} d_{2, u} \boldsymbol{a}_{T x, u}^{H} \boldsymbol{X} \boldsymbol{a}_{T x, u} \\
& \Phi\left(\phi_{u}, h_{R, u}\right)=\sigma_{u} \mathbb{R}\left\{h_{u} d_{1, u}\right\} \boldsymbol{a}_{T x, u}^{H} \boldsymbol{X} \boldsymbol{a}_{T x, u} \\
& \Phi\left(\phi_{u}, h_{I, u}\right)=\sigma_{u} \mathbb{I}\left\{h_{u} d_{1, u}\right\} \boldsymbol{a}_{T x, u}^{H} \boldsymbol{X} \boldsymbol{a}_{T x, u} \\
& \Phi\left(h_{R, u}, h_{R, u}\right)=\sigma_{u} d_{0, u} \boldsymbol{a}_{T x, u}^{H} \boldsymbol{X} \boldsymbol{a}_{T x, u} \\
& \Phi\left(h_{R, u}, h_{I, u}\right)=0 \\
& \Phi\left(h_{I, u}, h_{I, u}\right)=\sigma_{u} d_{0, u} \boldsymbol{a}_{T x, u}^{H} \boldsymbol{X} \boldsymbol{a}_{T x, u} \\
& \sum_{n=-N / 2}^{N / 2} \sum_{n=-N / 2} \\
& \quad \text { where, } \sigma_{u}=\frac{25 P_{T x, u} \xi_{u}}{N_{o}}, \boldsymbol{X}_{\tau}= \\
& \mid \boldsymbol{F}_{n} .
\end{aligned}
$$

\section{APPENDiX C}

\section{CONVEX REFORMULATION OF AOD CONSTRAinT}

In equation (13c) consider $u=\dot{\boldsymbol{a}}_{u}^{H} \boldsymbol{X}_{N} \dot{\boldsymbol{a}}_{u}, v=$ $\dot{\boldsymbol{a}}_{u}^{H} \boldsymbol{X}_{N} \boldsymbol{a}_{u}, w=\boldsymbol{a}_{u}^{H} \boldsymbol{X}_{N} \boldsymbol{a}_{u}$ and $k=\alpha_{\theta, u} \zeta_{\theta}$. Then,

$$
\begin{aligned}
& u-\frac{|v|^{2}}{w} \geq k \\
& w(u-k) \geq v^{H} v \\
& 4 w(u-k) \geq 4 v^{H} v \\
& 4 w(u-k)+w^{2}+(u-k)^{2} \geq(2|v|)^{2}+w^{2}+(u-k)^{2} \\
& ((u-k)+w)^{2} \geq(2|v|)^{2}+((u-k)-w)^{2} \\
& (u-k+w) \geq\left\|\left[\begin{array}{c}
2 \operatorname{Re}(v) \\
2 \operatorname{Im}(v) \\
(u-k-w)
\end{array}\right]\right\|_{2}
\end{aligned}
$$

\section{REFERENCES}

[1] F. Lemic, J. Martin, C. Yarp, D. Chan, V. Handziski, R. Brodersen, G. Fettweis, A. Wolisz, and J. Wawrzynek, "Localization as a feature of mmwave communication," in 2016 International Wireless Communications and Mobile Computing Conference (IWCMC), Sep. 2016, pp. 1033-1038.

[2] K. Wu, J. Xiao, Y. Yi, D. Chen, X. Luo, and L. M. Ni, "CSIbased indoor localization," IEEE Transactions on Parallel and Distributed Systems, vol. 24, no. 7, pp. 1300-1309, Jul. 2013.

[3] A. Shahmansoori, G. E. Garcia, G. Destino, G. Seco-Granados, and $\mathrm{H}$. Wymeersch, "5G position and orientation estimation through millimeter wave MIMO," in 2015 IEEE Globecom Workshops (GC Wkshps), Dec. 2015.

[4] A. Guerra, F. Guidi, and D. Dardari, "Single anchor localization and orientation performance limits using massive arrays: MIMO vs. beamforming," CoRR, vol. abs/1702.01670, 2017. [Online]. Available: http://arxiv.org/abs/1702.01670

[5] A. Shahmansoori, G. E. Garcia, G. Destino, G. Seco-Granados, and $\mathrm{H}$. Wymeersch, "Position and orientation estimation through millimeter-wave MIMO in $5 \mathrm{G}$ systems," IEEE Transactions on Wireless Communications, vol. 17, no. 3, pp. 1822-1835, Mar 2018.

[6] A. Alkhateeb, O. E. Ayach, G. Leus, and R. W. Heath, "Channel estimation and hybrid precoding for millimeter wave cellular systems," IEEE Journal of Selected Topics in Signal Processing, vol. 8, no. 5, pp. 831-846, Oct. 2014.

[7] A. Bazzi, D. T. M. Slock, L. Meilhac, and S. Panneerselvan, "A comparative study of sparse recovery and compressed sensing algorithms with application to AoA estimation," in 2016 IEEE 17th International Workshop on Signal Processing Advances in Wireless Communications (SPAWC), July 2016, pp. 1-5.

[8] S. Malla and G. Abreu, "Channel estimation in millimeter wave MIMO systems: Sparsity enhancement via reweighting," in 2016 International Symposium on Wireless Communication Systems (ISWCS), Sept 2016, pp. 230-234.

[9] N. Garcia, H. Wymeersch, and D. T. M. Slock, "Optimal robust precoders for tracking the $\mathrm{AoD}$ and $\mathrm{AoA}$ of a mm-wave path," CoRR, vol. abs/1703.10978, 2017. [Online]. Available: http://arxiv.org/abs/1703.10978

[10] R. Koirala, B. Denis, D. Dardari, and B. Uguen, "Localization bound based beamforming optimization for multicarrier mmwave MIMO," in 2017 14th Workshop on Positioning, Navigation and Communications (WPNC), Oct 2017, pp. 1-6.

[11] M. S. Lobo, L. Vandenberghe, S. Boyd, and H. Lebret, "Applications of second-order cone programming," Linear Algebra and its Applications, vol. 284, no. 1, pp. 193 - 228, 1998, international Linear Algebra Society (ILAS) Symposium on Fast Algorithms for Control, Signals and Image Processing.

[12] M. Grant and S. Boyd, "CVX: Matlab software for disciplined convex programming, version 2.1," http://cvxr.com/cvx, Mar 2014.

[13] F. Kelly, "Charging and rate control for elastic traffic," Transactions on Emerging Telecommunications Technologies, vol. 8, no. 1, pp. 33-37, 1997.

[14] Z. Q. Luo, W. K. Ma, A. M. C. So, Y. Ye, and S. Zhang, "Semidefinite relaxation of quadratic optimization problems," IEEE Signal Processing Magazine, vol. 27, no. 3, pp. 20-34, May 2010.

[15] G. Destino and H. Wymeersch, "On the trade-off between positioning and data rate for mm-wave communication," in 2017 IEEE International Conference on Communications Workshops (ICC Workshops), May 2017, pp. 797-802.

[16] T. S. Rappaport, G. R. MacCartney, M. K. Samimi, and S. Sun, "Wideband millimeter-wave propagation measurements and channel models for future wireless communication system design," IEEE Transactions on Communications, vol. 63, no. 9, pp. 3029-3056, Sep. 2015. 